\title{
Area of Ostectomy in Posterior Percutaneous Endoscopic Cervical Foraminotomy: Images and Mid-term Outcomes
}

\author{
Shu Nakamura, Mitsuto Taguchi \\ Department of Orthopedic Surgery, Aichi Spine Institute, Aichi, Japan
}

\begin{abstract}
Study Design: Retrospective.
Purpose: To analyze whether the cross-sectional area of the intervertebral foramen at the outermost edge of the resection site is associated with postoperative outcomes and whether our fluoroscopic method for determining the resection area is appropriate.

Overview of Literature: There is no consensus on the criteria for determining an optimal resection area to obtain sufficient decompression while maintaining intervertebral stability in posterior percutaneous endoscopic cervical foraminotomy. Previous reports have recommended a facet resection rate (FRR) of $\leq 50 \%$. Intervertebral foramen stenosis often extends to the exit zone. The crosssectional area of the intervertebral foramen is occasionally small at the outermost edge of the resection site. No report has analyzed whether these aspects are associated with postoperative outcomes.

Methods: Lateral margins of the resection area were set at lateral borders of the vertebral body on frontal fluoroscopic view. Because the percutaneous endoscope has a small diameter, surrounding structures can easily be identified using frontal view fluoroscopy to determine the resection area. FRRs were calculated from postoperative computed tomography images. The smallest crosssectional area of the intervertebral foramen around the lateral edge of the resection area (SALE) was measured and compared with clinical outcomes.

Results: The mean FRR was $41.7 \%$ at C5-C6 and 48.9\% at C6-C7. SALE was not correlated with clinical outcomes.

Conclusions: Residual stenosis in the lateral portion of the intervertebral foramen is weakly associated with postoperative outcomes. Our process achieved adequate FRRs and favorable postoperative outcomes, suggesting that our criteria for determining the resection area are appropriate.
\end{abstract}

Keywords: Foramen; Cervical; Percutaneous endoscope; Resection area

\section{Introduction}

In posterior percutaneous endoscopic cervical foraminotomy (P-PECF), an endoscope is placed close to the target to provide an excellent view in a minimally invasive man- ner [1,2]. Unlike anterior cervical decompression, posterior foraminotomy allows safe and efficient nerve root decompression and also preserves intervertebral mobility and prevents accelerated degeneration of the adjacent intervertebral discs [3-6]. However, in posterior foraminot-

Received Jan 13, 2017; Revised Mar 21, 2017; Accepted Mar 26, 2017

Corresponding author: Shu Nakamura

Department of Orthopedic Surgery, Aichi Spine Institute, 41 Gouhigashi, Takao, Fuso-cho, Niwa-gun, Aichi 480-0102, Japan

Tel: +81-587-92-3388, Fax: +81-587-92-3304, E-mail: nakamura@itoortho.or.jp 
omy, the spinous process restricts the direction of resection. Hence, the facet joint is also resected in proportion to the extent of intervertebral foramen decompression. Extensive facet joint resection may induce intervertebral instability. In three-dimensional finite element analysis models, facet joint resection by $50 \%$ results in $15 \%-18 \%$ increases in lateral bending and rotation [7]. In vitro models have demonstrated that facet joint resection by $>50 \%$ causes segmental hypermobility. Therefore, Zdeblick et al. [8] have recommended that facet joint resection and capsule stripping should be limited to $<50 \%$ of the facet. Nerve root compression is predominantly observed at the entrance zone of the intervertebral foramen, where the nerve is large. Decompression can be achieved by resecting the medial half of the facet joint [9]. Generally, in posterior foraminotomy, the medial side of the facet joint is removed while preserving the lateral side. However, in many cases, intervertebral foraminal stenosis extends to the exit zone. On resecting the facet joint by $\leq 50 \%$, the cross-sectional area of the intervertebral foramen at the lateral edge of the resection area cannot be sufficiently enlarged. The association between the cross-sectional area of the intervertebral foramen at the lateral edge of the resection area and postoperative outcomes remains unknown. This study aimed to elucidate this association. Although the resection area is difficult to determine using endoscopy, the percutaneous endoscope has a small diameter and does not interfere with surrounding structures on fluoroscopy. In the present study, we determined the resection area from the intraoperative frontal fluoroscopic view and examined whether this process is appropriate.

\section{Materials and Methods}

\section{General information}

In addition to conventional axial and sagittal views of magnetic resonance and computed tomography (CT) images, T2-weighted images were collected from the right and left sides at an oblique angle of $30^{\circ}$ from the sagittal plane for detailed preoperative examination of the intervertebral foramen (Fig. 1). This method provided approximate images of the cross-sectional area of the intervertebral foramen. Indications for P-PECF were radiculopathy due to cervical intervertebral foramen stenosis. This retrospective study included 104 patients (88 men and 16 women, mean age: 55.2 years) who underwent P-PECF

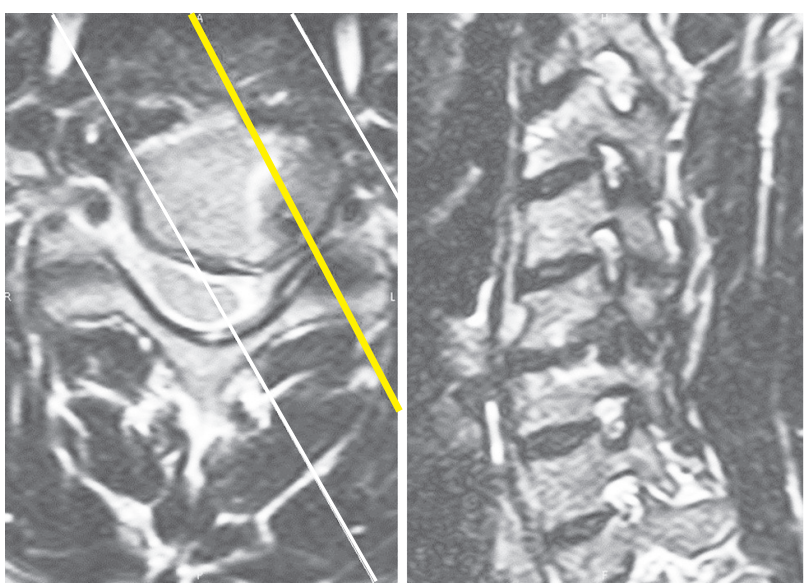

Fig. 1. T2-weighted magnetic resonance images were collected from the right and left sides at an oblique angle of $30^{\circ}$ (yellow line) from the sagittal plane for detailed preoperative examination of the intervertebral foramen.

performed by the first author between February 2011 and November 2015.

\section{Surgical method and follow-up}

A percutaneous full-endoscopic system was used, which comprised a light source, an endoscope, an irrigation channel, and a working channel. Surgery was performed under general anesthesia with patients in the prone position with a Relton-Hall frame and ProneView system (DUPACO, Oceanside, CA, USA). The neck was slightly flexed, and the head was fixed with tape. C-arm fluoroscopy was arranged to display frontal and lateral views of the cervical vertebrae. The operator stood contralateral to the affected side. A 7-mm-paramedian incision through the skin and fascia was made. An inferior oblique $\varphi 7.5$ sheath (ASAP, Umkirch, Germany) and a percutaneous endoscope (ASAP SES endoscope $\varphi 6.3 \mathrm{~mm}$ ) were inserted through the incisions after dilation using a dilator. The operator's elbows were placed on a stand to ensure the stability of the percutaneous endoscope. Ostectomy was performed using a percutaneous endoscopic surgical drill with diamond burrs of 3 and $2 \mathrm{~mm}$ in diameter. Sharp points at ostectomy site edges were removed using a curved curette (Fig. 2). The drain was removed the following day. Patients were ambulated at $3 \mathrm{~h}$ after surgery and discharged the following day. They wore a soft neck collar for 1 week and were scheduled to visit the outpatient clinic at 1, 6, 12, and 24 months postoperatively. 


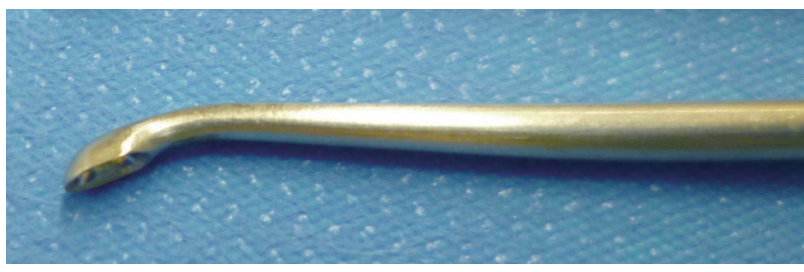

Fig. 2. Sharp points at the edges of the ostectomy site were removed using a curved curette.

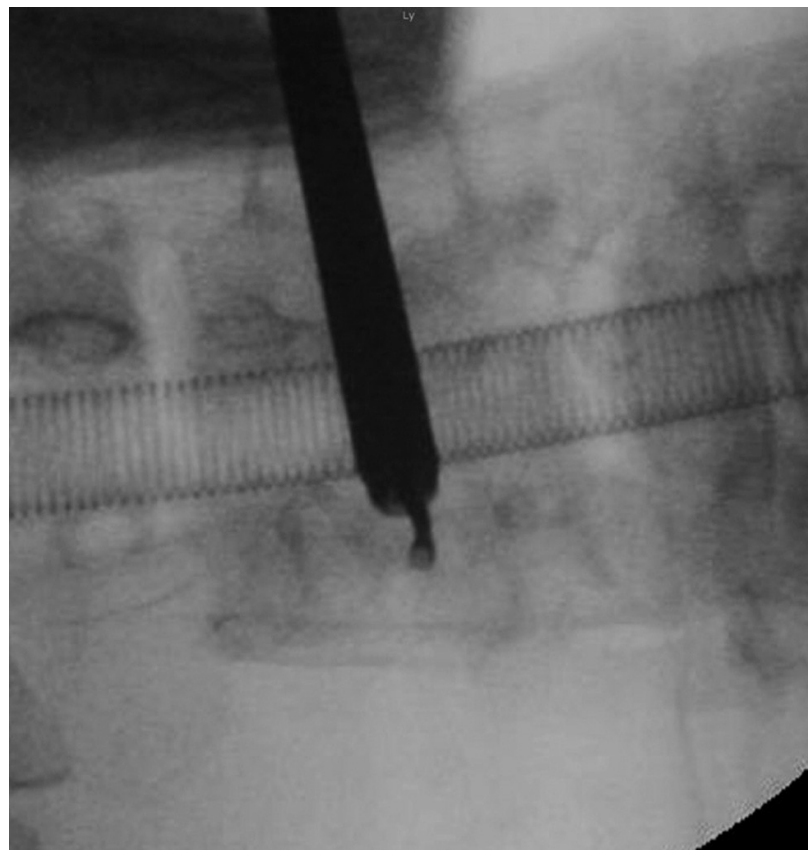

Fig. 3. Lateral aspect of the facet joint was resected sufficiently beyond the lateral borders of the vertebral body under frontal fluoroscopic view. The lateral borders of the vertebral body are often difficult to identify on fluoroscopic images and should be located based on the positional relationship with the pedicle at the cranial and caudal sides on preoperative radiographs.

\section{Facet joint resection area}

Although the perineural membrane was preserved, nerve root relief was visible under hydraulic pressure. Cranial and caudal sides of the facet joint were resected until sufficient decompression was endoscopically confirmed. The lateral aspect of the facet joint was sufficiently resected beyond lateral borders of the vertebral body under frontal fluoroscopic view. Lateral borders of the vertebral body are often difficult to identify on fluoroscopic images and should be located based on the positional relationship with the pedicle at cranial and caudal sides on preoperative radiographs (Fig. 3).
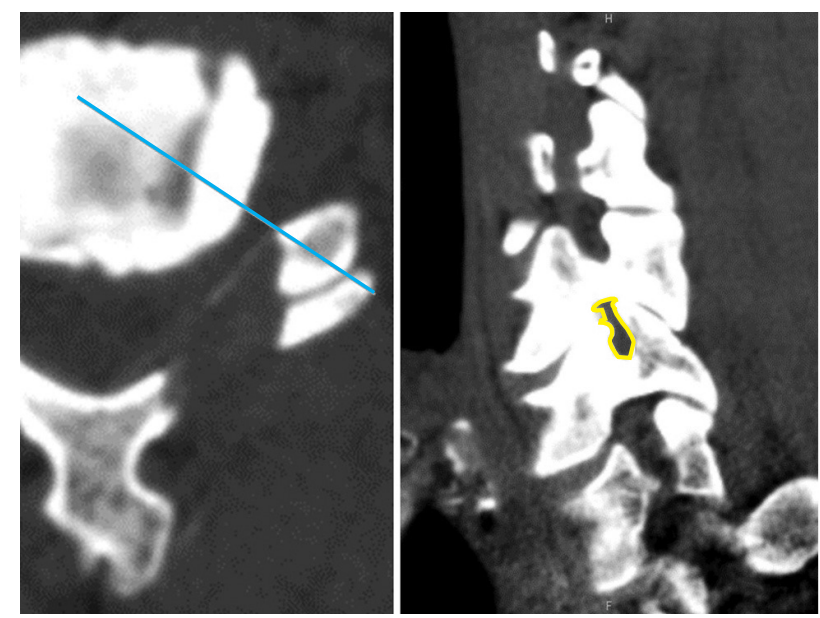

Fig. 4. Postoperative computed tomography images were reconstructed to obtain several cross-sectional images vertical to the longitudinal axis of the intervertebral foramen at the operated vertebral level. The smallest cross-sectional area (yellow line) of the intervertebral foramen around the lateral edge (blue line) of the resection area (SALE) was selected and measured.

\section{Postoperative evaluations}

Using the POP-net web server (ImageONE Co., Tokyo, Japan) of a Digital Imaging and Communications in Medicine viewer, postoperative $\mathrm{CT}$ images were reconstructed to obtain several cross-sectional images vertical to the longitudinal axis of the intervertebral foramen at the operated vertebral level. The smallest cross-sectional area of the intervertebral foramen around the lateral edge of the resection area (SALE) was selected and measured (Fig. 4).

Patients completed self-administered questionnaires on preoperative and postoperative symptoms. Clinical outcomes were determined based on visual analog scale (VAS) scores for cervical and upper limb symptoms and the general symptom remnant $(\mathrm{GR}=$ current general symptom VAS/preoperative general symptom VAS $\times 10$ ), which reflects the severity of residual symptoms, including cervical and upper limb symptoms. GR scores of $0-3$ were considered as excellent, $0-6$ as responsive to treatment, and $\geq 7$ as poor.

From axial CT images of the middle level of the facet joint, the preoperative facet length (FL) and postoperative facet length (poFL) were measured. The facet resection rate (FRR) was calculated using the following formula: (FL-poFL)/FL $\times 100 \%$ (Fig. 5). Hypothetical FRRs (hFRRs) were calculated for an area extending $2 \mathrm{~mm}$ laterally from outer edges of the vertebral body (Fig. 6). Actual FRRs (aFRRs) were calculated immediately after surgery. All 

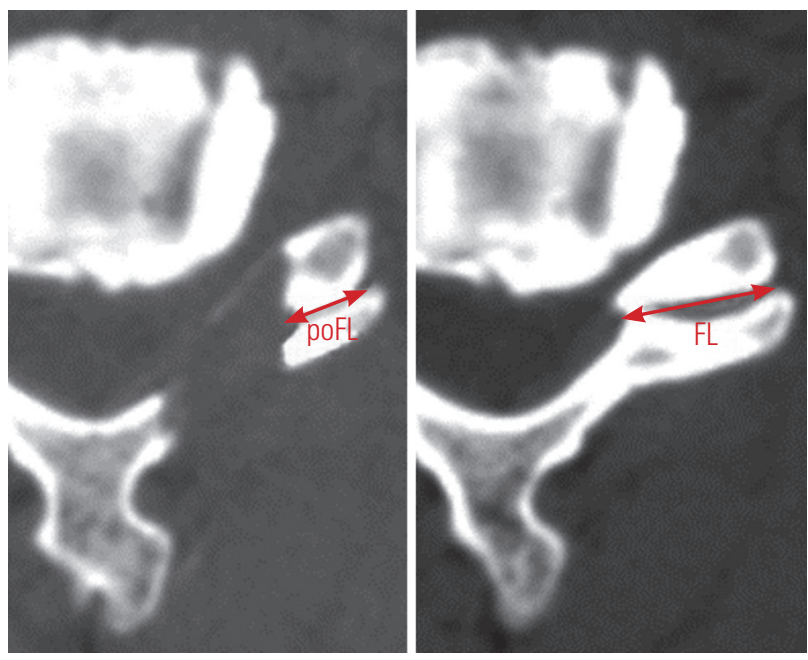

Fig. 5. From axial computed tomography images of the middle level of the facet joint, the preoperative facet length (FL) and postoperative facet length $(\mathrm{poFL})$ were measured. The facet resection rate $(\mathrm{FRR})=(\mathrm{FL}-$ poFL)/FL.

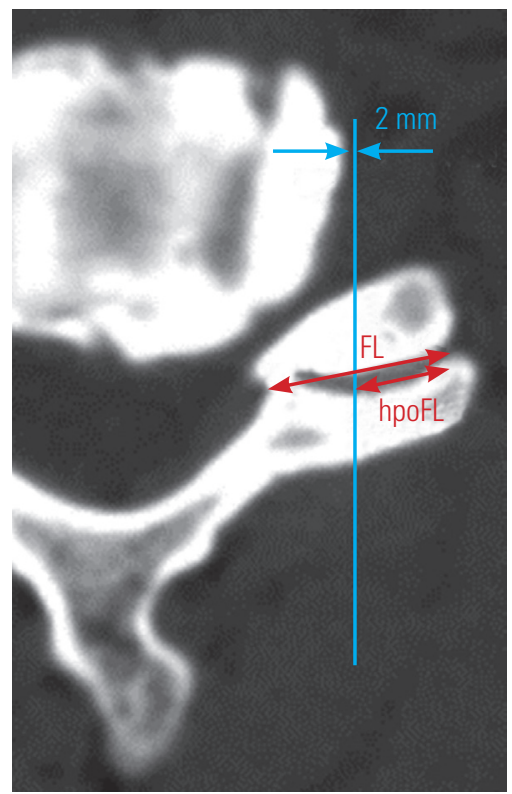

Fig. 6. Hypothetical facet resection rate (hFRRs) were calculated for an area extending for $2 \mathrm{~mm}$ laterally (blue line) from the outer edges of the vertebral body. FL, facet length; hpoFL, hypothetical postoperative facet length; $h F R R=(F L-h p o F L) / F L$.

measurements were performed by an orthopedist who was blinded to the clinical outcome.

\section{Statistical analysis}

We calculated the Pearson's correlation coefficient to assess the association between GR and SALE. Differences between the excellent and poor outcome groups were analyzed using unpaired Student's $t$-test or Fisher's exact test. A $p$-value of $<0.05$ was considered significant.

\section{Results}

Of the 104 patients, 10 had lesions in C4-C5, 38 in C5C6, 54 in C6-C7, and two in C7-T1. Dural tear or apparent root injury did not occur during surgery in any patient. Excluding 20 patients enrolled during the early study period, the mean operative time for the remaining patients was 76.7 minutes. No patient developed spondylolisthesis after surgery. Data on postoperative outcomes were obtained from 92 patients (mean follow-up period, 10.1 months [range, 1-24 months]). Excellent outcomes (GR; current general symptom, 0-3) were achieved in $66.3 \%$ of them (61 patients, the excellent outcome group). Patients responsive to P-PECF (GR, 0-6), including those in the excellent outcome group, accounted for $87.0 \%$ of the total patients ( 80 patients, the responsive group). Poor outcomes ( $\mathrm{GR} \geq 7)$ were observed in $13.0 \%$ patients (12 patients, the poor outcome group). Reasons for poor outcomes (including multiple reasons in some patients) were multiple operations on the neck in four patients (33\%), concurrent stenosis at another site in seven (58\%), unknown in two (17\%), diagnosis-related problems in one $(8 \%)$, and insufficient decompression in one (8\%) who was enrolled in the early study period.

Among patients undergoing single-level P-PECF who did not undergo any other surgical procedure for the operated vertebra, SALE was measured in 82 patients with GR data. The mean SALE was $0.58 \mathrm{~cm}^{2}$ (range, $0.14-1.18$ $\mathrm{cm}^{2}$ ). No correlation was observed between GR and SALE $(r=0.0785, p=0.241)$. A comparison between 57 patients in the excellent outcome group (GR, 0-3) and nine in the poor outcome group $(\mathrm{GR} \geq 7)$ revealed that the mean SALE was slightly larger in the former $\left(0.59 \mathrm{~cm}^{2}\right)$ than in the latter $\left(0.48 \mathrm{~cm}^{2}\right)$, but this difference was not significant (unpaired $t$-test, $p=0.088$ ).

A comparison of GRs obtained within 6 months after surgery and GRs obtained at 1-2 years after surgery indicated that no patient had GR worsened by $\geq 3$ points. No patient underwent reoperation at the same site because of deterioration.

VAS scores for preoperative cervical and upper limb symptoms were 6.68 and 6.14 in the excellent outcome group and 6.38 and 7.00 in the poor outcome group, re- 
spectively. Although VAS scores for preoperative upper limb symptoms were higher in the poor outcome group than in the excellent outcome group, no significant difference was observed in VAS scores for cervical or upper limb symptoms ( $p=0.378$ and 0.201 , respectively).

Among consecutive surgical patients enrolled in the late study period, $\mathrm{P}-\mathrm{PECF}$ was performed at $\mathrm{C} 5-\mathrm{C} 6$ in 20 patients and $\mathrm{C} 6-\mathrm{C} 7$ in 20 patients. For $\mathrm{C} 5-\mathrm{C} 6$, the mean hFRR was $37.6 \%$ (range, $25.8 \%-52.4 \%$ ) and the mean aFRR was $41.7 \%$ (range, $13.7 \%-57.7 \%$ ). For C6-C7, the mean hFRR was $46.4 \%$ (range, $28.1 \%-59.0 \%$ ) and the mean aFRR was $48.9 \%$ (range, 38.9\%-56.7\%). A correlation was observed between aFRRs and hFRRs $(r=0.48161$, $\left.p=0.826 \times 10^{-3}\right)$.

Eight patients underwent CT immediately after surgery and at $\geq 1$ year after surgery. In all patients, CT revealed reductions in the size of the resection area (at the ostectomy site area shown from the dorsal side using threedimensional CT). Annual reduction rates were $40.4 \%$ (range, 15.4\%-73.8\%) for the resection area, 24.8\% (range, $5.3 \%-50.8 \%$ ) for SALE, and $37.7 \%$ (range, $8.0 \%-86.9 \%$ ) for the width of the facet joint resection area. Despite these reductions, no patient suffered a relapse of symptoms.

\section{Discussion}

\section{Setting the resection area}

In P-PECF, the perineural membrane is compressed by perfusion pressure. This makes the nerve root relief clearly visible while preserving the perineural membrane, thereby reducing the risk of severe bleeding. Because cranial and caudal edges of the nerve root are visible, determining whether the decompressed area is sufficient at cranial and caudal sides is easy. However, determining whether lateral sides of the decompressed area are sufficient based on the morphology of the nerve root in the endoscopic view is difficult. Unlike the dura, the root is not distended too much even when it is loosened; therefore, constriction at the root is unlikely to be distinguished. In addition, the foramen is a narrow space and contains no gap on the dorsal side even under loose conditions. Therefore, checking whether there is sufficient room is not possible from the dorsal view. Although adequate facet joint resection is necessary to decompress the nerve root, the resection area is limited because of the risk of intervertebral stability.
Zdeblick et al. [8] have reported that instability slightly increases with an FRR of up to $50 \%$ but markedly increases with an FRR of $75 \%$. They have further indicated that the joint capsule plays an important role in intervertebral stability [8]. No consensus has been reached on the diagnostic criteria for determining an optimal resection area to obtain both adequate decompression and intervertebral stability. Although the resection area width is determined based on preoperative measurement, intraoperatively determining the amount of resected facet joint is difficult. One report has stated that using the width of a drill burr as a measure, an area extending for 3-4 $\mathrm{mm}$ from the inner edge of the facet joint can be resected under the endoscopic view [10]. However, this method is ambiguous. As ostectomy progresses during surgery, the landmarks are removed, and accurate measurement is consequently difficult.

Due to its small diameter, a percutaneous endoscope does not interfere in the viewing of surrounding structures on the intraoperative frontal fluoroscopic view. Thus, in the present study, an area extending sufficiently beyond the outer edges of the vertebral body was decompressed using the outer edges or pedicle on the intraoperative frontal fluoroscopic view as a landmark. The distance to the outer edge of the facet joint was checked to determine the resection area. This process was easy to execute during surgery, the decompressed area appeared to be necessary and sufficient, and favorable outcomes were obtained. Poor postoperative outcomes were associated with multiple rounds of cervical surgery and stenosis at other sites. Insufficient decompression was not a major cause. The severity of preoperative symptoms did not seem to affect postoperative outcomes.

The mean aFRR was $41.7 \%$ at $\mathrm{C} 5-\mathrm{C} 6$ and $48.9 \%$ at C6C7, suggesting that the setting methods for the resection area were appropriate. Although there were patients in whom the facet joint was resected by $>50 \%$, it is assumed that an FRR of $>50 \%$ does not cause any problems with intervertebral stability because of postoperative bone regeneration. On preoperative CT images, hFRRs were calculated to be $37.6 \%$ at $\mathrm{C} 5-\mathrm{C} 6$ and $46.4 \%$ at C6-C7. hFRRs varied depending on the vertebral level and also varied markedly among patients. Furthermore, a correlation was observed between aFRRs and hFRRs. It may be preferable to individually set the resection rate for each patient according to the morphology of the lesion, similar to the process used in the present study. Despite the lack of evi- 
dence of intervertebral instability on images, excessively large resection areas lead to reduced stability and risk, accelerating intervertebral disc degeneration. However, it is assumed that resection by a little more than $50 \%$ does not cause any problem because of subsequent articular regeneration. Outcomes tend to be more favorable with a larger SALE, and the resection area reduces with time. Thus, it seems better to prioritize sufficient facet joint decompression over its preservation. Furthermore, ostectomy performed with a drill whose burr tip is spherical leaves sharp points on the ventral edges of the resection area. Removing these points with a curved curette may reduce nerve irritation.

\section{SALE and clinical outcomes}

FRR is used as an index to reflect the facet joint resection area. Because FRR is difficult to calculate using a threedimensional model, in most cases, it is calculated from two-dimensional images. Thus, FRR is not precise and cannot be regarded as a direct index of decompression. Although one report on the association between FRR and clinical outcomes has been published [10], the association between SALE and clinical outcomes remains unknown. In the present study, no correlation was found between clinical outcomes and SALE in the early or late study period and no significant difference in SALE was observed between the excellent and poor outcome groups. These findings are attributable to the importance of nerve root decompression in the medial portion of the intervertebral foramen, where the nerve is large [9]. Intervertebral foramen restenosis progressed from the lateral aspect because of bone regeneration at the ostectomy site after surgery; however, the symptoms did not relapse. This may be associated with the absence of a correlation between SALE and clinical outcomes. Although limiting FRRs to $\leq 50 \%$ to prevent intervertebral instability may result in insufficient decompression at the lateral aspect of the intervertebral foramen due to a reduced resection area, it seems that clinical outcomes are not greatly affected.

\section{Limitations of the present study}

This study was retrospective, and the dropout rate during follow-up was relatively high (11.5\%). Moreover, the follow-up period was not long. In this study, we investigated how the resection area relates to clinical results; therefore, we believe that it is reasonable to assess mid-term results rather than long-term results, which are greatly affected by the natural course.

\section{Conclusions}

The criteria used to determine the resection area on the intraoperative frontal fluoroscopic view are appropriate and useful for determining the resection area and achieving an adequate FRR and favorable postoperative outcomes. Residual stenosis at the lateral aspect of the intervertebral foramen is weakly associated with postoperative outcomes, therefore limiting FRRs to prevent intervertebral instability does not seem to interfere with favorable clinical outcomes.

\section{Conflict of Interest}

No potential conflict of interest relevant to this article was reported.

\section{ORCID}

Shu Nakamura: https://orcid.org/0000-0002-8015-7571

\section{References}

1. Ruetten S, Komp M, Merk H, Godolias G. Fullendoscopic cervical posterior foraminotomy for the operation of lateral disc herniations using 5.9-mm endoscopes: a prospective, randomized, controlled study. Spine (Phila Pa 1976) 2008;33:940-8.

2. Ruetten S, Komp M, Merk H, Godolias G. A new full-endoscopic technique for cervical posterior foraminotomy in the treatment of lateral disc herniations using 6.9-mm endoscopes: prospective 2-year results of 87 patients. Minim Invasive Neurosurg 2007;50:219-26.

3. Caglar YS, Bozkurt M, Kahilogullari G, et al. Keyhole approach for posterior cervical discectomy: experience on 84 patients. Minim Invasive Neurosurg 2007;50:7-11.

4. Clarke MJ, Ecker RD, Krauss WE, McClelland RL, Dekutoski MB. Same-segment and adjacent-segment disease following posterior cervical foraminotomy. J Neurosurg Spine 2007;6:5-9.

5. Jagannathan J, Sherman JH, Szabo T, Shaffrey CI, 
Jane JA. The posterior cervical foraminotomy in the treatment of cervical disc/osteophyte disease: a single-surgeon experience with a minimum of 5 years' clinical and radiographic follow-up. J Neurosurg Spine 2009;10:347-56.

6. Hilibrand AS, Balasubramanian K, Eichenbaum M, et al. The effect of anterior cervical fusion on neck motion. Spine (Phila Pa 1976) 2006;31:1688-92.

7. Chen BH, Natarajan RN, An HS, Andersson GB. Comparison of biomechanical response to surgical procedures used for cervical radiculopathy: posterior keyhole foraminotomy versus anterior foraminotomy and discectomy versus anterior discectomy with fu- sion. J Spinal Disord 2001;14:17-20.

8. Zdeblick TA, Zou D, Warden KE, McCabe R, Kunz D, Vanderby R. Cervical stability after foraminotomy: a biomechanical in vitro analysis. J Bone Joint Surg Am 1992;74:22-7.

9. Tanaka N, Fujimoto Y, An HS, Ikuta Y, Yasuda M. The anatomic relation among the nerve roots, intervertebral foramina, and intervertebral discs of the cervical spine. Spine (Phila Pa 1976) 2000;25:286-91.

10. Kim CH, Kim KT, Chung CK, et al. Minimally invasive cervical foraminotomy and diskectomy for laterally located soft disk herniation. Eur Spine J 2015;24:3005-12. 